\title{
Article \\ On the Unique Solvability of Incomplete Cauchy Type Problems for a Class of Multi-Term Equations with the Riemann-Liouville Derivatives
}

\author{
Vladimir E. Fedorov ${ }^{1,2,+}+$ (D), Wei-Shih $\mathrm{Du}^{3, *,+(\mathbb{D})}$ and Mikhail M. Turov ${ }^{1,+(\mathbb{D})}$ \\ 1 Department of Mathematical Analysis, Mathematics Faculty, Chelyabinsk State University, \\ Kashirin Brothers St., 129, 454001 Chelyabinsk, Russia; kar@csu.ru (V.E.F.); turov_m_m@mail.ru (M.M.T.) \\ 2 Laboratory of Functional Materials, South Ural State University (National Research University), Lenin Av., 76, \\ 454080 Chelyabinsk, Russia \\ 3 Department of Mathematics, National Kaohsiung Normal University, Kaohsiung 82444, Taiwan \\ * Correspondence: wsdu@mail.nknu.edu.tw \\ $\dagger$ V.E.F., W.-S.D. and M.M.T. contributed equally to this work.
}

check for

updates

Citation: Fedorov, V.E.; Du, W.-S.; Turov, M.M. On the Unique Solvability of Incomplete Cauchy Type Problems for a Class of Multi-Term Equations with the Riemann-Liouville Derivatives. Symmetry 2022, 14, 75. https:// doi.org/10.3390/sym14010075

Academic Editor: Ioan Raşa

Received: 4 December 2021 Accepted: 23 December 2021 Published: 5 January 2022

Publisher's Note: MDPI stays neutral with regard to jurisdictional claims in published maps and institutional affiliations.

Copyright: () 2022 by the authors Licensee MDPI, Basel, Switzerland. This article is an open access article distributed under the terms and conditions of the Creative Commons Attribution (CC BY) license (https:// creativecommons.org/licenses/by/ $4.0 /)$.

\begin{abstract}
Incomplete Cauchy-type problems are considered for linear multi-term equations solved with respect to the highest derivative in Banach spaces with fractional Riemann-Liouville derivatives and with linear closed operators at them. Some new existence and uniqueness theorems for solutions are presented explicitly and the analyticity of the solutions of the homogeneous equations are also shown. The asymmetry of the Cauchy-type problem under study is expressed in the presence of a so-called defect, which shows the number of lower-order initial conditions that should not be set when setting the problem. As applications, our abstract results are used in the study of a class of initial-boundary value problems for multi-term equations with Riemann-Liouville derivatives in time and with polynomials of a self-adjoint elliptic differential operator with respect to spatial variables.
\end{abstract}

Keywords: Riemann-Liouville fractional derivative; incomplete Cauchy-type problem; multi-term fractional differential equation; defect of Cauchy-type problem; analytic solution in a sector; initialboundary value problem

MSC: 35R11; 34G10; 34A08

\section{Introduction}

A linear multi-term fractional equation

$$
D_{t}^{\alpha} z(t)=\sum_{j=1}^{m-1} A_{j} D_{t}^{\alpha-m+j} z(t)+\sum_{l=1}^{n} B_{l} D_{t}^{\alpha_{l}} z(t)+\sum_{s=1}^{r} C_{s} J_{t}^{\beta_{s}} z(t)+f(t)
$$

is investigated in this work, where $D_{t}^{\gamma}$ is the Riemann-Liouville derivative with $\gamma>0$, $J_{t}^{\gamma}$ is the Riemann-Liouville integral, $m-1<\alpha \leq m \in \mathbb{N}, 0<\alpha_{1}<\alpha_{2}<\cdots<\alpha_{n}<\alpha$, $m_{l}-1<\alpha_{l} \leq m_{l} \in \mathbb{N}, \alpha_{l}-m_{l} \neq \alpha-m, l=1,2, \ldots, n, \beta_{1}>\beta_{2}>\cdots>\beta_{r} \geq 0$, $f \in C((0, T) ; D) \cap L_{1}(0, T ; D)$, operators $A_{j}, j=1,2, \ldots, m-1, B_{l}, l=1,2, \ldots, n, C_{s}$, $s=1,2, \ldots, r$, are linear and closed with dense domains in the Banach space $\mathcal{Z}$, and the intersection $D$ of which are also dense in the space $\mathcal{Z}$ and equipped with the sum of the norms of the graphs of these operators.

In the case $\mathcal{Z}=\mathbb{R}$ issues of the unique solvability of the Cauchy-type problem

$$
D_{t}^{\alpha-m+k} z(0)=z_{k}, k=0,1, \ldots, m-1,
$$

for Equation (1) were investigated in [1-7] under various constraints on $\alpha_{n}$. It is shown in [6] that this problem is uniquely solvable if and only if $\alpha_{n}<\alpha-m+1$. In [8], it is shown that for Equation (1), generally speaking, only the incomplete Cauchy-type problem 


$$
D_{t}^{\alpha-m+k} z(0)=z_{k}, k=m^{*}, m^{*}+1, \ldots, m-1,
$$

can be solved. Here $m^{*}$ is a number defined by a set of orders of derivatives $\alpha, \alpha_{1}, \alpha_{2}, \ldots$, $\alpha_{n}$, it is called the defect of the Cauchy type problem. If a solution of (1) and (2) exists, then $D_{t}^{\alpha-m+k} z(0)=0$ for $k=0,1, \ldots, m^{*}-1$. This is the asymmetry of the studied Cauchy-type problem. In [8], the unique solvability of the incomplete Cauchy-type problem (1) and (3) is proved in the case of bounded operators $A_{j}, j=1,2, \ldots, m-1, B_{l}, l=1,2, \ldots, n, C_{s}$, $s=1,2, \ldots, r$, at $f \in C((0, T) ; \mathcal{Z}) \cap L_{1}(0, T ; \mathcal{Z})$.

In this paper, a class $\mathcal{A}_{\alpha}^{n, r}\left(\theta_{0}, a_{0}\right)$ of tuples of linear closed, densely-defined operators is introduced into consideration, $\theta_{0} \in(\pi / 2, \pi), a_{0} \geq 0$. It is shown that belonging of operators to this class implies the existence of a unique classical solution of the problem (1) and (3), which at $f \equiv 0$ can be analytically continued into the sector $\{t \in \mathbb{C}:|\arg t|<$ $\left.\theta_{0}-\pi / 2, t \neq 0\right\}$. Proposed class $\mathcal{A}_{\alpha}^{n, r}\left(\theta_{0}, a_{0}\right)$ generalizes the class of operators $\mathcal{A}_{\alpha}\left(\theta_{0}, a_{0}\right)$ (see $[9,10])$, into which it passes if $A_{j}=B_{l}=C_{s}=0, j=1,2, \ldots, m-1, l=1,2, \ldots, n$, $s=1,2, \ldots, r-1, \beta_{r}=0$, and $C_{r}$ is a linear closed operator with a dense domain in $\mathcal{Z}$. The unique solvability of the Cauchy-type problem for linear and nonlinear equations with the Riemann-Liouville derivative and with an operator from $\mathcal{A}_{\alpha}\left(\theta_{0}, a_{0}\right)$ in the right-hand side was studied in [11-15]. We also note the works [16,17], in which the initial problems for multi-term equations of form (1) with the Gerasimov-Caputo derivative were investigated.

This paper is organized as follows. In Section 2, the definitions for the fractional Riemann-Liouville derivative and integral, for the classical solution of the considered incomplete Cauchy-type problem and for the defect $m^{*}$ of the Cauchy-type problem are given. In Section 3 , the class $\mathcal{A}_{\alpha}^{n, r}\left(\theta_{0}, a_{0}\right)$ of tuples of operators is defined and the existence and uniqueness of a classical solution for problem (1) and (3) is proved in the case of

$$
\left(A_{1}, A_{2}, \ldots, A_{m-1}, B_{1}, B_{2}, \ldots, B_{n}, C_{1}, C_{2}, \ldots, C_{r}\right) \in \mathcal{A}_{\alpha}^{n, r}\left(\theta_{0}, a_{0}\right)
$$

and $f \equiv 0$. The solution is presented explicitly using the Laplace transform technique. In Section 4, this result is extended to the case of inhomogeneous Equation (1) with $f \in C((0, T) ; D) \cap L_{1}(0, T ; D)$. Finally, in Section 5 , the obtained results are used for the statement and the investigation of the unique solvability of a class of initial-boundary value problems for equations with several Riemann-Liouville derivatives in time and with polynomials of a self-adjoint elliptic differential operator with respect to spatial variables. A nontrivial example illustrating our results is also given. In contrast to the results of work [8] on the problem (1) and (3) with bounded operators, in this case the equations are considered, in which the degrees of polynomials at lower fractional derivatives in time can exceed the degree of the polynomial at the highest time-derivative.

\section{Incomplete Cauchy Type Problem and Its Defect}

Let $\mathcal{Z}$ be a Banach space, $\mathcal{L}(\mathcal{Z})$ denote the Banach space of linear bounded operators in $\mathcal{Z}$, and by $\mathcal{C l}(\mathcal{Z})$ the set of linear closed operators with dense domains in $\mathcal{Z}$ be denoted, $\mathbb{R}_{+}:=\{a \in \mathbb{R}: a>0\}, \overline{\mathbb{R}}_{+}:=\mathbb{R}_{+} \cup\{0\}, h: \mathbb{R}_{+} \rightarrow \mathcal{Z}$. For $\beta>0$ let $g_{\beta}(t):=t^{\beta-1} / \Gamma(\beta)$ at $t>0$,

$$
J_{t}^{\beta} h(t):=\left(g_{\beta} * h\right)(t):=\int_{0}^{t} g_{\beta}(t-s) h(s) d s=\frac{1}{\Gamma(\beta)} \int_{0}^{t}(t-s)^{\beta-1} h(s) d s .
$$

By $J_{t}^{0}$, we denote the identity operator. Let $\alpha>0, m$ be the smallest integer, which is greater or equal $\alpha, D_{t}^{m}$ be the usual derivative of the order $m \in \mathbb{N}$, and $D_{t}^{\alpha}$ be the fractional Riemann-Liouville derivative, i.e., $D_{t}^{\alpha} h(t)=D_{t}^{m} J_{t}^{m-\alpha} h(t)$. At $\beta<0$, we will use the notation $D_{t}^{\beta} h(t):=J_{t}^{-\beta} h(t)$.

By $\mathfrak{L}[h]$, we denote the Laplace transform of a function $h: \mathbb{R}_{+} \rightarrow \mathcal{Z}$, and by $\mathfrak{L}^{-1}[H]$ the inverse Laplace transform of a function $H: \Omega \rightarrow \mathcal{Z}, \Omega \supset\{\mu \in \mathbb{C}: \operatorname{Re} \mu>\omega\}$ at some 
$\omega \in \mathbb{R}$, is denoted. The Laplace transforms of the fractional Riemann-Liouville integral and the fractional Riemann-Liouville derivative, respectively satisfy the following equalities

$$
\mathfrak{L}\left[J_{t}^{\alpha} h\right](\lambda)=\lambda^{-\alpha} \mathfrak{L}[h](\lambda)
$$

and

$$
\mathfrak{L}\left[D_{t}^{\alpha} h\right](\lambda)=\lambda^{\alpha} \mathfrak{L}[h](\lambda)-\sum_{k=0}^{m-1} \lambda^{m-1-k} D_{t}^{\alpha-m+k} h(0),
$$

where $D_{t}^{\alpha-m+k} h(0):=\lim _{t \rightarrow 0+} D_{t}^{\alpha-m+k} h(t)$. For further details, see [18] and references therein.

Let $m-1<\alpha \leq m \in \mathbb{N}, 0<\alpha_{1}<\alpha_{2}<\cdots<\alpha_{n}<\alpha, m_{l}-1<\alpha_{l} \leq m_{l} \in \mathbb{N}$, $\alpha_{l}-m_{l} \neq \alpha-m, l=1,2, \ldots, n, \beta_{1}>\beta_{2}>\cdots>\beta_{r} \geq 0$. Denote

$$
\underline{\alpha}:=\max \left\{\alpha_{l}: l \in\{1,2, \ldots, n\}, \alpha_{l}-m_{l}<\alpha, m\right\},
$$

and

$$
\bar{\alpha}:=\max \left\{\alpha_{l}: l \in\{1,2, \ldots, n\}, \alpha_{l}-m_{l}>\alpha-m\right\} .
$$

We define

$$
\underline{m}=\lceil\underline{\alpha}\rceil \text { and } \bar{m}=\lceil\bar{\alpha}\rceil,
$$

where $\lceil\cdot\rceil$ indicates the ceiling function.

Remark 1. Note that for integers $\alpha_{l}$ we have $\alpha_{l}=m_{l}$, so they always fall into the second group, provided that $\alpha$ is not an integer, otherwise the second group is empty; in the first group there are fractional numbers only. Therefore, by construction it is always $\underline{\alpha}<\underline{m}, \bar{m}<m$ (otherwise there will be $\alpha_{l}$, greater than $\alpha$ ). If any of the sets, which determine the numbers $\bar{\alpha}, \underline{\alpha}$, is empty, we put by definition the corresponding value $\underline{m}$ or $\bar{m}$ equal to zero.

In this paper, we consider a linear multi-term fractional differential equation

$$
D_{t}^{\alpha} z(t)=\sum_{j=1}^{m-1} A_{j} D_{t}^{\alpha-m+j} z(t)+\sum_{l=1}^{n} B_{l} D_{t}^{\alpha_{l}} z(t)+\sum_{s=1}^{r} C_{s} J_{t}^{\beta_{s}} z(t), \quad t \in \mathbb{R}_{+}
$$

where $A_{j} \in \mathcal{C l}(\mathcal{Z}), j=1,2, \ldots, m-1, B_{l} \in \mathcal{C l}(\mathcal{Z}), l=1,2, \ldots, n, C_{s} \in \mathcal{C l} l(\mathcal{Z}), s=$ $1,2, \ldots, r$, i.e., closed linear operators with domains $D_{A_{1}}, D_{A_{2}}, \ldots, D_{A_{m-1}}, D_{B_{1}}, D_{B_{2}}, \ldots, D_{B_{n}}$, $D_{C_{1}}, D_{C_{2}}, \ldots, D_{C_{r}}$, respectively. A function $z: \mathbb{R}_{+} \rightarrow \mathcal{Z}$ is called a solution of Equation (4), if $J_{t}^{m-\alpha} z \in C^{m}\left(\mathbb{R}_{+} ; \mathcal{Z}\right), J_{t}^{m-\alpha} z \in C^{j}\left(\mathbb{R}_{+} ; D_{A_{j}}\right), j=1,2, \ldots, m-1, J_{t}^{m_{l}-\alpha_{l}} z \in C^{m_{l}}\left(\mathbb{R}_{+} ; D_{B_{l}}\right)$, $l=1,2, \ldots, n, J_{t}^{\beta_{s}} z \in C\left(\mathbb{R}_{+} ; D_{C_{s}}\right), s=1,2, \ldots, r$, and equality (4) holds for $t \in \mathbb{R}_{+}$.

For the equation, consider the Cauchy-type problem:

$$
D_{t}^{\alpha-m+k} z(0)=z_{k}, \quad k=0,1, \ldots, m-1 .
$$

The following statements were proved in [8].

Corollary 1. If $\alpha>\alpha_{l}, \alpha_{l}-m_{l}>\alpha-m$ for some $l \in\{1,2, \ldots, n\}$ and for some $k \in$ $\left\{0,1, \ldots, m_{l}-1\right\} \lim _{t \rightarrow 0+} D_{t}^{\alpha_{l}-m_{l}+k} z(t) \neq 0$, then there is no solution to problem (4) and (5).

Corollary 2. If $\alpha>\alpha_{l}, \alpha_{l}-m_{l}<\alpha-m$ for some $l \in\{1,2, \ldots, n\}$ and for some $k \in$ $\left\{0,1, \ldots, m_{l}-1\right\} \lim _{t \rightarrow 0+} D_{t}^{\alpha_{l}-m_{l}+k} z(t) \neq 0$, then there is no solution to problem (4) and (5).

Corollary 3. If $\underline{\alpha}>1$ and $z_{k} \neq 0$ for any $k \in\{0,1, \ldots, \underline{m}-2\}$, then there is no solution to problem (4) and (5). 
Corollary 4. If $\bar{\alpha}>0$ and $z_{k} \neq 0$ for any $k \in\{0,1, \ldots, \bar{m}-1\}$, then there is no solution of problem (4) and (5).

By applying results presented above, one can easily see that there exists a solution of problem (4) and (5), only if

$$
D_{t}^{\alpha-m+k} z(0)=0 \text { for } k=0,1, \ldots, m^{*}-1
$$

and

$$
D_{t}^{\gamma} z(0)=0 \text { for } \gamma \in \Lambda \text {, }
$$

where $\Lambda:=\left\{\alpha_{1}-1, \alpha_{1}-2, \ldots, \alpha_{1}-m_{1}, \alpha_{2}-1, \alpha_{2}-2, \ldots, \alpha_{n}-m_{n}\right\}$. Therefore, for Equation (4) it makes sense to consider only, generally speaking, the incomplete Cauchytype problem

$$
D_{t}^{\alpha-m+k} z(0)=z_{k} \quad \text { for } k=m^{*}, m^{*}+1, \ldots, m-1,
$$

where $m^{*}:=\max \{\underline{m}-1, \bar{m}\}$ is the so-called defect of the Cauchy-type problem [8], which is determined by the set of numbers $\alpha, \alpha_{1}, \alpha_{2}, \ldots, \alpha_{n}$ and characterizes the number of the lower initial conditions omitted in the incomplete problem. It is not difficult to make sure that $0 \leq m^{*} \leq m-1$ (see [8]).

\section{Analytic in a Sector Solutions of a Homogeneous Equation}

Let $A_{1}, A_{2}, \ldots, A_{m-1}, B_{1}, B_{2}, \ldots, B_{n}, C_{1}, C_{2}, \ldots, C_{r}$ be closed linear operators with domains $D_{A_{1}}, D_{A_{2}}, \ldots, D_{A_{1}}, D_{B_{1}}, D_{B_{2}}, \ldots, D_{B_{n}}, D_{C_{1}}, D_{C_{2}}, \ldots, D_{C_{r}}$ respectively. Denote:

$$
D:=\bigcap_{j=1}^{m-1} D_{A_{j}} \cap \bigcap_{l=1}^{n} D_{B_{l}} \cap \bigcap_{s=1}^{r} D_{C_{s}}
$$

and

$$
R_{\lambda}:=\left(\lambda^{\alpha} I-\sum_{j=1}^{m-1} \lambda^{\alpha-m+j} A_{j}-\sum_{l=1}^{n} \lambda^{\alpha_{l}} B_{l}-\sum_{s=1}^{r} \lambda^{-\beta_{s}} C_{s}\right)^{-1}: \mathcal{Z} \rightarrow D
$$

We supply the set $D$ with the norm

$$
\|\cdot\|_{D}=\|\cdot\|_{\mathcal{Z}}+\sum_{j=1}^{m-1}\left\|A_{j} \cdot\right\|_{\mathcal{Z}}+\sum_{l=1}^{n}\left\|B_{l} \cdot\right\|_{\mathcal{Z}}+\sum_{s=1}^{r}\left\|C_{s} \cdot\right\|_{\mathcal{Z}}
$$

with respect to which $D$ is a Banach space, since it is the intersection of the Banach spaces $D_{A_{1}}, D_{A_{2}}, \ldots, D_{A_{m-1}}, D_{B_{1}}, D_{B_{2}}, \ldots, D_{B_{n}}, D_{C_{1}}, D_{C_{2}}, \ldots, D_{C_{r}}$ with the corresponding graph norms.

Definition 1. A tuple of operators $\left(A_{1}, A_{2}, \ldots, A_{m-1}, B_{1}, B_{2}, \ldots, B_{n}, C_{1}, C_{2}, \ldots, C_{r}\right)$ belongs to the class $\mathcal{A}_{\alpha}^{n, r}\left(\theta_{0}, a_{0}\right)$ for some $\theta_{0} \in(\pi / 2, \pi), a_{0} \geq 0$, if

(i) $D$ is dense in $\mathcal{Z}$;

(ii) For all $\lambda \in S_{\theta_{0}, a_{0}}:=\left\{\mu \in \mathbb{C}:\left|\arg \left(\mu-a_{0}\right)\right|<\theta_{0}\right\}, p=0,1, \ldots, m-1$, operators:

$$
R_{\lambda} \cdot\left(I-\sum_{j=p+1}^{m-1} \lambda^{j-m} A_{j}\right) \in \mathcal{L}(\mathcal{Z})
$$

exist;

(iii) For any $\theta \in\left(\pi / 2, \theta_{0}\right), a>a_{0}$, there exists such a $K(\theta, a)$ that for all $\lambda \in S_{\theta, a}, p=$ $0,1, \ldots, m-1$, we have

$$
\left\|R_{\lambda} \cdot\left(I-\sum_{j=p+1}^{m-1} \lambda^{j-m} A_{j}\right)\right\|_{\mathcal{L}(\mathcal{Z})} \leq \frac{K(\theta, a)}{|\lambda-a||\lambda|^{\alpha-1}}
$$


Remark 2. It immediately follows from condition (ii) that $R_{\lambda} \in \mathcal{L}(\mathcal{Z})$ (take $p=m-1$ ) and $R_{\lambda} A_{p} \in \mathcal{L}(\mathcal{Z})$ for all $p=0,1, \ldots, m-1$, since

$$
R_{\lambda} \cdot\left(I-\sum_{j=p+1}^{m-1} \lambda^{j-m} A_{j}\right)-R_{\lambda} \cdot\left(I-\sum_{j=p}^{m-1} \lambda^{j-m} A_{j}\right)=\lambda^{p-m} R_{\lambda} A_{p} .
$$

Note also that the inequality in (iii) at $p=m-1$ has the form

$$
\left\|R_{\lambda}\right\|_{\mathcal{L}(\mathcal{Z})} \leq \frac{K(\theta, a)}{|\lambda-a||\lambda|^{\alpha-1}}
$$

Remark 3. When $\beta_{r}=0$, the condition $\left(0, \ldots, 0, C_{r}, 0, \ldots, 0\right) \in \mathcal{A}_{\alpha}^{n, r}\left(\theta_{0}, a_{0}\right)$ is satisfied, if and only if $C_{r} \in \mathcal{A}_{\alpha}\left(\theta_{0}, a_{0}\right)$ (see works [9-15]), at $\alpha=1$ such an operator is called sectorial [19,20].

Theorem 1. Let $m-1<\alpha \leq m \in \mathbb{N}, 0<\alpha_{1}<\alpha_{2}<\cdots<\alpha_{n}<\alpha, m_{l}-1<$ $\alpha_{l} \leq m_{l} \in \mathbb{N}, \alpha_{l}-m_{l} \neq \alpha-m, l=1,2, \ldots, n, \beta_{1}>\beta_{2}>\cdots>\beta_{r} \geq 0, A_{j} \in$ $\mathcal{C l}(\mathcal{Z}), j=1,2, \ldots, m-1, B_{l} \in \mathcal{C} l(\mathcal{Z}), l=1,2, \ldots, n, C_{s} \in \mathcal{C l}(\mathcal{Z}), s=1,2, \ldots, r$, $\left(A_{1}, A_{2}, \ldots, A_{m-1}, B_{1}, B_{2}, \ldots, B_{n}, C_{1}, C_{2}, \ldots, C_{r}\right) \in \mathcal{A}_{\alpha}^{n, r}\left(\theta_{0}, a_{0}\right), z_{k} \in D, k=m^{*}, m^{*}+1, \ldots$, $m-1$. Then there exists a unique solution to problem (4) and (6), and it has the form

$$
z(t)=\sum_{p=m^{*}}^{m-1} Z_{p}(t) z_{p}
$$

where:

$$
Z_{p}(t)=\frac{1}{2 \pi i} \int_{\Gamma_{\varepsilon, r_{0}}} \lambda^{m-\alpha} R_{\lambda}\left(\lambda^{\alpha-1-p} I-\sum_{j=p+1}^{m-1} \lambda^{\alpha-m-1+j-p} A_{j}\right) e^{\lambda t} d \lambda,
$$

$\Gamma_{\varepsilon, r_{0}}:=\Gamma_{\varepsilon, r_{0}}^{+} \cup \Gamma_{\varepsilon, r_{0}}^{-} \cup \Gamma_{\varepsilon, r_{0}}^{0}, \Gamma_{\varepsilon, r_{0}}^{0}:=\left\{\lambda \in \mathbb{C}:\left|\lambda-a_{0}-\varepsilon\right|=r_{0}>0, \arg \lambda \in\left(-\theta_{0}+\varepsilon, \theta_{0}-\varepsilon\right)\right\}$, $\Gamma_{\varepsilon, r_{0}}^{ \pm}:=\left\{\lambda \in \mathbb{C}: \arg \left(\lambda-a_{0}-\varepsilon\right)= \pm\left(\theta_{0}-\varepsilon\right),\left|\lambda-a_{0}-\varepsilon\right| \in\left[r_{0}, \infty\right)\right\}, \varepsilon \in\left(0, \theta_{0}-\pi / 2\right)$, $r_{0}>0$. Moreover, the solution can be analytically continued in the sector $\{t \in \mathbb{C}:|\arg t|<$ $\left.\theta_{0}-\pi / 2, t \neq 0\right\}$.

Proof. Note that by virtue of Definition 1 for $\lambda \in \Gamma_{\varepsilon, r_{0}}$,

$$
\left\|\lambda^{m-\alpha} R_{\lambda}\left(\lambda^{\alpha-1-p} I-\sum_{j=p+1}^{m-1} \lambda^{\alpha-m-1+j-p} A_{j}\right)\right\|_{\mathcal{L}(\mathcal{Z})} \leq \frac{K\left(\theta_{0}-\varepsilon, a_{0}+\varepsilon\right)|\lambda|^{m-\alpha-p}}{\left|\lambda-a_{0}-\varepsilon\right|}
$$

Consider the integral defining $Z_{p}(t)$ for $t>0$, on the part $\Gamma_{\varepsilon, r_{0}}^{+}$of the contour $\Gamma_{\varepsilon, r_{0}}$, take $\lambda=a_{0}+\varepsilon+r e^{i\left(\theta_{0}-\varepsilon\right)}$ and get an upper bound for the norm of this integral at $p=0$, we then obtain

$$
\begin{aligned}
c_{1} e^{\left(a_{0}+\varepsilon\right) t} \int_{r_{0}}^{+\infty} r^{m-\alpha-1} e^{r \cos \left(\theta_{0}-\varepsilon\right) t} d r & \leq c_{1} e^{\left(a_{0}+\varepsilon\right) t} \int_{t r_{0} c_{2}}^{+\infty} \frac{\tau^{m-\alpha-1} e^{-\tau}}{t^{m-\alpha} c_{2}^{m-\alpha}} d \tau \\
& \leq \frac{c_{1} c_{2}^{\alpha-m} \Gamma(m-\alpha) e^{\left(a_{0}+\varepsilon\right) t}}{t^{m-\alpha}}
\end{aligned}
$$

where $c_{2}=\left|\cos \left(\theta_{0}-\varepsilon\right)\right|$. For $p=1,2, \ldots, m-1$, we get

$$
c_{1} e^{\left(a_{0}+\varepsilon\right) t} \int_{r_{0}}^{+\infty} r^{m-\alpha-1-p} e^{-c_{2} r t} d r \leq \frac{c_{1} r_{0}^{m-\alpha-p} e^{\left[\left(a_{0}+\varepsilon\right)-c_{2} r_{0}\right] t}}{\alpha-m+p} .
$$


On $\Gamma_{\varepsilon, r_{0}}^{-}$, we have similar inequalities. Such an estimate for the norm of the integral on the part $\Gamma_{\varepsilon, r_{0}}^{0}$ of the contour is obtained after substituting $\lambda=a_{0}+\varepsilon+r_{0} e^{i \varphi}$ and have the form at $p=0,1, \ldots, m-1$,

$$
c_{1} r_{0}^{m-\alpha-1-p} e^{\left(a_{0}+\varepsilon\right) t} \int_{-\theta_{0}+\varepsilon}^{\theta_{0}-\varepsilon} e^{t r_{0} \cos \varphi} d \varphi \leq 2 \pi c_{1} r_{0}^{m-\alpha-1-p} e^{\left(a_{0}+\varepsilon+r_{0}\right) t} .
$$

As a result,

$$
\left\|Z_{0}(t)\right\|_{\mathcal{L}(\mathcal{Z})} \leq \frac{C e^{\left(a_{0}+\varepsilon\right) t}}{t^{m-\alpha}}, \quad\left\|Z_{p}(t)\right\|_{\mathcal{L}(\mathcal{Z})} \leq C e^{\left(a_{0}+\mathcal{E}+r_{0}\right) t}
$$

for $p=1,2, \ldots, m-1$, and to the functions $Z_{p}, p=0,1, \ldots, m-1$, the Laplace transform can be applied, since $m-\alpha<1$. In addition, it follows from estimates (7) that the families of operators $\left\{Z_{p}(t): t>0\right\}, p=0,1, \ldots, m-1$, can be analytically continued into the sector $\left\{t \in \mathbb{C}:|\arg t|<\theta_{0}-\pi / 2, t \neq 0\right\}$, since $\varepsilon \in\left(0, \theta_{0}-\pi / 2\right)$ is arbitrary.

Take $\mu \in \mathbb{C}$ from the right side of the contour $\Gamma_{\varepsilon, r_{0}}$, we have

$$
\left\|\frac{\lambda^{m-\alpha} R_{\lambda}}{\mu-\lambda} \cdot\left(\lambda^{\alpha-1-p} I-\sum_{j=p+1}^{m-1} \lambda^{\alpha-m-1+j-p} A_{j}\right)\right\|_{\mathcal{L}(\mathcal{Z})} \leq \frac{K\left(\theta_{0}-\varepsilon, a_{0}+\varepsilon\right)|\lambda|^{m-\alpha-p}}{\left|\lambda-a_{0}-\varepsilon\right||\mu-\lambda|} .
$$

Since $2+p+\alpha-m>1$, the function

$$
\begin{aligned}
\mathfrak{L}\left[Z_{p}\right](\mu) & =\frac{1}{2 \pi i} \int_{\Gamma_{\varepsilon, r_{0}}} \frac{\lambda^{m-\alpha} R_{\lambda}}{\mu-\lambda} \cdot\left(\lambda^{\alpha-1-p} I-\sum_{j=p+1}^{m-1} \lambda^{\alpha-m-1+j-p} A_{j}\right) d \lambda \\
& =\mu^{m-1-p} R_{\mu}\left(I-\sum_{j=p+1}^{m-1} \mu^{j-m} A_{j}\right)
\end{aligned}
$$

is analytic in the part of the plane $\mathbb{C}$-bounded from the left by the contour $\Gamma_{\varepsilon, r_{0}}$. Since $\varepsilon$ and $r_{0}$ can be taken arbitrarily small, then $\mathfrak{L}\left[Z_{p}\right](\mu)$ are analytic in $S_{\theta_{0}, a_{0}}$ at $p=0,1, \ldots, m-1$. Using this fact, it is not difficult to show that the value of $Z_{p}(t)$ does not depend on the choice of parameters $\varepsilon \in\left(0, \theta_{0}-\pi / 2\right), r_{0}>0$.

For $p=0,1, \ldots, m-1, p \geq m^{*}, z_{p} \in D$, we have

$$
\begin{gathered}
\mathfrak{L}\left[J_{t}^{m-\alpha} Z_{p}\right](\mu)=\mu^{\alpha-1-p} R_{\mu}\left(I-\sum_{j=p+1}^{m-1} \mu^{j-m} A_{j}\right), \\
J_{t}^{m-\alpha} Z_{p}(t) z_{p}=\frac{1}{2 \pi i} \int_{\Gamma_{\varepsilon, r_{0}}} R_{\lambda}\left(\lambda^{\alpha} I-\sum_{j=p+1}^{m-1} \lambda^{\alpha-m+j} A_{j}\right) \frac{e^{\lambda t} d \lambda}{\lambda^{p+1}} z_{p} \\
=\frac{1}{2 \pi i} \int_{\Gamma_{\varepsilon, r_{0}}} \frac{e^{\lambda t} d \lambda}{\lambda^{p+1}} z_{p}+\frac{1}{2 \pi i} \int_{\Gamma_{\varepsilon, R}} R_{\lambda}\left(\sum_{j=1}^{p} \lambda^{\alpha-m+j} A_{j}+\sum_{l=1}^{n} \lambda^{\alpha_{l}} B_{l}+\sum_{s=1}^{r} \lambda^{-\beta_{s}} C_{s}\right) \frac{e^{\lambda t} d \lambda}{\lambda^{p+1}} z_{p}
\end{gathered}
$$

and

$$
\begin{aligned}
& \left\|\int_{\Gamma_{\varepsilon, R}} R_{\lambda}\left(\sum_{j=1}^{p} \lambda^{\alpha-m+j} A_{j}+\sum_{l=1}^{n} \lambda^{\alpha_{l}} B_{l}+\sum_{s=1}^{r} \lambda^{-\beta_{s}} C_{s}\right) \frac{d \lambda}{\lambda^{p+1}} z_{p}\right\|_{\mathcal{Z}} \\
& \left.\leq \underset{\Gamma_{\varepsilon, R}}{ } \int_{j=1}^{p}|\lambda|^{-m+j}\left\|A_{j} z_{p}\right\|_{\mathcal{Z}}+\sum_{l=1}^{n}|\lambda|^{\alpha_{l}-\alpha}\left\|B_{l} z_{p}\right\|_{\mathcal{Z}}+\sum_{s=1}^{r}|\lambda|^{-\beta_{s}-\alpha}\left\|C_{s} z_{p}\right\|_{\mathcal{Z}}\right) \frac{|d \lambda|}{|\lambda|^{p+1}} \rightarrow 0,
\end{aligned}
$$


when $R \rightarrow \infty$, since $p+1+m-p=m+1>1, p+1+\alpha-\alpha_{l} \geq 1+\alpha-\alpha_{l}>1$, $p+1+\alpha+\beta_{s}>1$. Therefore, $J_{t}^{m-\alpha} Z_{0}(0) z_{0}=z_{0}$ for $z_{0} \in D$ and $J_{t}^{m-\alpha} Z_{p}(0) z_{p}=0$ for $z_{p} \in D, p=1,2, \ldots, m-1$.

Next, let $m^{*}=0$, i.e., $\underline{m} \leq 1$ and $\bar{m}=0$. This means that $\alpha_{l}-m_{l}<\alpha-m$ and $\alpha_{l}<1$ for all $l=1,2, \ldots, n$. Then for $\alpha>1$ we have $\alpha-\alpha_{l}>1$ and for $z_{0} \in D$,

$$
\begin{aligned}
\mathfrak{L}\left[D_{t}^{\alpha-m+1} Z_{0}(t) z_{0}\right](\mu) & =\mu^{\alpha} R_{\mu}\left(z_{0}-\sum_{j=1}^{m-1} \mu^{j-m} A_{j} z_{0}\right)-z_{0} \\
& =R_{\mu}\left(\sum_{l=1}^{n} \mu^{\alpha_{l}} B_{l}+\sum_{s=1}^{r} \mu^{-\beta_{s}} C_{s}\right) z_{0}
\end{aligned}
$$

and

$$
D_{t}^{\alpha-m+1} Z_{0}(t) z_{0}=\frac{1}{2 \pi i} \int_{\Gamma_{\varepsilon, r_{0}}} R_{\lambda}\left(\sum_{l=1}^{n} \lambda^{\alpha_{l}} B_{l}+\sum_{s=1}^{r} \lambda^{-\beta_{s}} C_{s}\right) e^{\lambda t} z_{0} d \lambda,
$$

therefore, $D_{t}^{\alpha-m+1} Z_{0}(0) z_{0}=0$. Continuing this process, at the $m$ th step we get

$$
D_{t}^{\alpha-1} Z_{0}(t) z_{0}=\frac{1}{2 \pi i} \int_{\Gamma_{\varepsilon, r_{0}}} R_{\lambda}\left(\sum_{l=1}^{n} \lambda^{\alpha_{l}+m-2} B_{l}+\sum_{s=1}^{r} \lambda^{-\beta_{s}+m-2} C_{s}\right) e^{\lambda t} z_{0} d \lambda
$$

and

$$
D_{t}^{\alpha-1} Z_{0}(0) z_{0}=0,
$$

since $m_{l} \leq 1, \alpha_{l}+m-2-\alpha<m_{l}-2 \leq-1$.

Let $m^{*} \leq p$, i.e., $m \leq p+1, \bar{m} \leq p$. Then $\alpha_{l}<p+1$ for $\alpha_{l}-m_{l}<\alpha-m$, and also $\alpha_{l} \leq p$ for $\alpha_{l}-m_{l}>\alpha-m$. We get at $p=1,2, \ldots, m-1, q=1,2, \ldots, p, z_{p} \in D$ such that

$$
\begin{gathered}
D_{t}^{\alpha-m+q} Z_{p}(t) z_{p}=\frac{1}{2 \pi i} \int_{\Gamma_{\varepsilon, r_{0}}} \frac{e^{\lambda t} d \mu}{\lambda^{p-q+1}} z_{p} \\
+\frac{1}{2 \pi i} \int_{\Gamma_{\varepsilon, r_{0}}} R_{\lambda}\left(\sum_{j=1}^{p} \lambda^{\alpha-m+j} A_{j}+\sum_{l=1}^{n} \lambda^{\alpha_{l}} B_{l}+\sum_{s=1}^{r} \lambda^{-\beta_{s}} C_{s}\right) \frac{e^{\lambda t} z_{p} d \lambda}{\lambda^{p-q+1}} .
\end{gathered}
$$

Therefore, at $q<p$, since $p-q+1 \geq 2$, we obtain $D_{t}^{\alpha-m+q} Z_{p}(0) z_{p}=0$. From here it can be seen that $D_{t}^{\alpha-m+p} Z_{p}(0) z_{p}=z_{p}$ due to the inequalities $m-p+1>1, \alpha-\alpha_{l}+1>1$, $\alpha+\beta_{s}+1>1$.

For $p \leq m-2$ and $z_{p} \in D$, we have

$$
\begin{aligned}
\mathfrak{L}\left[D_{t}^{\alpha-m+p+1} Z_{p}(t) z_{p}\right](\mu) & =\mu^{\alpha} R_{\mu}\left(z_{p}-\sum_{j=p+1}^{m-1} \mu^{j-m} A_{j} z_{p}\right)-z_{p} \\
& =R_{\mu}\left(\sum_{j=1}^{p} \mu^{\alpha-m+j} A_{j}+\sum_{l=1}^{n} \mu^{\alpha_{l}} B_{l}+\sum_{s=1}^{r} \mu^{-\beta_{s}} C_{s}\right) z_{p}
\end{aligned}
$$

and

$$
D_{t}^{\alpha-m+p+1} Z_{p}(t) z_{p}=\frac{1}{2 \pi i} \int_{\Gamma_{\varepsilon, r_{0}}} R_{\lambda}\left(\sum_{j=1}^{p} \lambda^{\alpha-m+j} A_{j}+\sum_{l=1}^{n} \lambda^{\alpha_{l}} B_{l}+\sum_{s=1}^{r} \lambda^{-\beta_{s}} C_{s}\right) e^{\lambda t} z_{p} d \lambda .
$$


In this case, $j-m \leq p-m \leq-2$, for $\alpha_{l}-m_{l}<\alpha-m$,

$$
\alpha-\alpha_{l}>m-m_{l} \geq m-\underline{m} \geq m-(p+1) \geq m-(m-2)-1=1,
$$

is executed and for $\alpha_{l}-m_{l}>\alpha-m$, the inequalities are valid $\alpha-\alpha_{l} \geq \alpha-p \geq \alpha-m+2>1$. Hence, $\alpha>1, D_{t}^{\alpha-m+p+1} Z_{p}(0) z_{p}=0$. Continuing the process, we get at $p \leq m-3$, $q=p+2, p+3, \ldots, m-1$,

$$
D_{t}^{\alpha-m+q} Z_{p}(t) z_{p}=\frac{1}{2 \pi i} \int_{\Gamma_{\varepsilon, r_{0}}} \lambda^{q-p-1} R_{\lambda}\left(\sum_{j=1}^{p} \lambda^{\alpha-m+j} A_{j}+\sum_{l=1}^{n} \lambda^{\alpha_{l}} B_{l}+\sum_{s=1}^{r} \lambda^{-\beta_{s}} C_{s}\right) e^{\lambda t} z_{p} d \lambda,
$$

since $j-m+q-p-1 \leq q-m-1 \leq 2$, for $\alpha_{l}-m_{l}<\alpha-m$, we have

$$
p+1-q+\alpha-\alpha_{l}>p+2-m+m-m_{l}=p+2-m_{l} \geq 1,
$$

and for $\alpha_{l}-m_{l}>\alpha-m$, the inequalities

$$
p+1-q+\alpha-\alpha_{l} \geq p+2-m+\alpha-p=2-m+\alpha>1
$$

hold. Since $p+1-q<0$, it follows that $\alpha>1, D_{t}^{\alpha-m+q} Z_{p}(0) z_{p}=0$.

Further, by virtue of Corollaries 1 and 2, we obtain

$$
\lim _{t \rightarrow 0+} D_{t}^{\alpha_{l}-m_{l}+k} Z_{p}(t) z_{p}=0 \text { for } z_{p} \in D \text { and } l=1,2, \ldots, n,
$$

which implies

$$
\begin{aligned}
\mathfrak{L} & {\left[D_{t}^{\alpha} Z_{p}(t) z_{p}\right](\mu)=\mu^{\alpha} \mathfrak{L}\left[Z_{p}(t) z_{p}\right](\mu)-\mu^{m-1-p_{z}} z_{p} } \\
& =\mu^{\alpha} R_{\mu}\left(\mu^{m-1-p_{z}} z_{p}-\sum_{j=p+1}^{m-1} \mu^{j-1-p} A_{j} z_{p}\right)-\mu^{m-1-p_{z}} \\
& =\left(\sum_{j=1}^{m-1} \mu^{\alpha-m+j} A_{j}+\sum_{l=1}^{n} \mu^{\alpha_{l}} B_{l}+\sum_{s=1}^{r} \mu^{-\beta_{s}} C_{s}\right) \mu^{m-1-p} R_{\mu}\left(z_{p}-\sum_{j=p+1}^{m-1} \mu^{j-m} A_{j} z_{p}\right) \\
& -\sum_{j=p+1}^{m-1} \mu^{j-1-p} A_{j} z_{p} \\
& =\mathfrak{L}\left[\sum_{j=1}^{m-1} A_{j} D_{t}^{\alpha-m+j} Z_{p}(t) z_{p}+\sum_{l=1}^{n} B_{l} D_{l}^{\alpha_{l}} Z_{p}(t) z_{p}+\sum_{s=1}^{r} C_{s} J_{t}^{\beta_{s}} Z_{p}(t) z_{p}\right](\mu) .
\end{aligned}
$$

By acting of the inverse Laplace transform, we get that $Z_{p}(t) z_{p}$ at $z_{p} \in D$ is a solution of Equation (4) for $t>0$ and $p=m^{*}, m^{*}+1, \ldots, m-1$.

Let $z_{1}(t)$ and $z_{2}(t)$ be two solutions of problem (4) and (6) on $\mathbb{R}_{+}$. Fix $T>0$, then $y(t)=z_{1}(t)-z_{2}(t)$ is a solution of problem (4) and (6) with $z_{p}=0, p=m^{*}, m^{*}+1, \ldots, m-$ 1 , on the interval $(0, T)$. Let us define the function $y$ as zero for $t \in[T,+\infty)$. Such a function is bounded and is also a solution to this problem for all $t>0$, except maybe $t=T$. Note that by virtue of Corollaries 1 and 2, $\lim _{t \rightarrow 0+} D_{t}^{\alpha_{l}-m_{l}+k} y(t)=0$ for $l=1,2, \ldots, n$. Let us act by the Laplace transform on both parts of the equality

$$
D_{t}^{\alpha} y(t)=\sum_{j=1}^{m-1} A_{j} D_{t}^{\alpha-m+j} y(t)+\sum_{l=1}^{n} B_{l} D_{t}^{\alpha_{l}} y(t)+\sum_{s=1}^{r} C_{s} J_{t}^{\beta_{l}} y(t)
$$


and hence we get

$$
\begin{aligned}
\mathfrak{L}\left[D_{t}^{\alpha} y\right](\mu) & =\mu^{\alpha} \mathfrak{L}[y](\mu) \\
& =\mathfrak{L}\left[\sum_{j=1}^{m-1} A_{j} D_{t}^{\alpha-m+j} y+\sum_{l=1}^{n} B_{l} D_{t}^{\alpha_{l}} y+\sum_{s=1}^{r} C_{s} J_{t}^{\beta_{s}} y\right](\mu) \\
& =\left(\sum_{j=1}^{m-1} \mu^{\alpha-m+j} A_{j}+\sum_{l=1}^{n} \mu^{\alpha_{l}} B_{l}+\sum_{s=1}^{r} \mu^{-\beta_{s}} C_{s}\right) \mathfrak{L}[y](\mu),
\end{aligned}
$$

which deduces

$$
\left(\mu^{\alpha} I-\sum_{j=1}^{m-1} \mu^{\alpha-m+j} A_{j}-\sum_{l=1}^{n} \mu^{\alpha_{l}} B_{l}-\sum_{s=1}^{r} \mu^{-\beta_{s}} C_{s}\right) \mathfrak{L}[y](\mu) \equiv 0
$$

and $\mathfrak{L}[y](\mu) \equiv 0$ at $\mu \in S_{\theta_{0}, a_{0}}$. Therefore, we obtain $z_{1}(t)-z_{2}(t)=y(t) \equiv 0$ for $t \in(0, T)$. Since $T>0$ can be chosen arbitrarily large, $z_{1}(t)=z_{2}(t)$ for all $t>0$.

Remark 4. From equality (8), it can be seen that for $z_{p} \in D$,

$$
Z_{p}(t) z_{p}=J_{t}^{p} Z_{0}(t) z_{p}+\frac{1}{2 \pi i} \int_{\Gamma_{\varepsilon, r_{0}}} R_{\mu} \sum_{j=1}^{p} \mu^{j-p-1} A_{j} z_{p} e^{\mu t} d \mu
$$

\section{Inhomogeneous Equation}

Consider the linear inhomogeneous multi-term fractional equation:

$$
D_{t}^{\alpha} z(t)=\sum_{j=1}^{m-1} A_{j} D_{t}^{\alpha-m+j} z(t)+\sum_{l=1}^{n} B_{l} D_{t}^{\alpha_{l}} z(t)+\sum_{s=1}^{r} C_{s} J_{t}^{\beta_{s}} z(t)+f(t)
$$

with initial conditions

$$
D_{t}^{\alpha-m+k} z(0)=0, k=m^{*}, m^{*}+1, \ldots, m-1 .
$$

A function $z:(0, T) \rightarrow \mathcal{Z}$ is called a solution of (9) and (10), if $J_{t}^{m-\alpha} z \in C^{m}((0, T) ; \mathcal{Z}) \cap$ $C^{m-1}([0, T) ; \mathcal{Z}), J_{t}^{m-\alpha} z \in C^{j}\left((0, T) ; D_{A_{j}}\right), j=1,2, \ldots, m-1, J_{t}^{m_{l}-\alpha_{l}} z \in C^{m_{l}}\left((0, T) ; D_{B_{l}}\right)$, $l=1,2, \ldots, n, J_{t}^{\beta_{s}} z \in C\left((0, T) ; D_{C_{s}}\right), s=1,2, \ldots, r$, and equalities (9) for $t \in(0, T)$ and (10) are satisfied. Recall that

$$
Z_{m-1}(t):=\frac{1}{2 \pi i} \int_{\Gamma_{\varepsilon, r_{0}}}\left(\lambda^{\alpha} I-\sum_{j=1}^{m-1} \lambda^{\alpha-m+j} A_{j}-\sum_{l=1}^{n} \lambda^{\alpha_{l}} B_{l}-\sum_{s=1}^{r} \lambda^{\beta_{s}} C_{s}\right)^{-1} e^{\lambda t} d \lambda, t>0,
$$

and introduce the operators

$$
V_{\kappa}(t):=\frac{1}{2 \pi i} \int_{\Gamma_{\varepsilon, r_{0}}} \lambda^{\kappa} R_{\lambda} e^{\lambda t} d \lambda, \quad t>0, \quad \kappa \in \mathbb{R} .
$$

Theorem 2. Let $m-1<\alpha \leq m \in \mathbb{N}, 0<\alpha_{1}<\alpha_{2}<\cdots<\alpha_{n}<\alpha, m_{l}-1<$ $\alpha_{l} \leq m_{l} \in \mathbb{N}, \alpha_{l}-m_{l} \neq \alpha-m, l=1,2, \ldots, n, \beta_{1}>\beta_{2}>\cdots>\beta_{r} \geq 0, A_{j} \in$ $\mathcal{C} l(\mathcal{Z}), j=1,2, \ldots, m-1, B_{l} \in \mathcal{C} l(\mathcal{Z}), l=1,2, \ldots, n, C_{s} \in \mathcal{C l} l(\mathcal{Z}), s=1,2, \ldots, r$, 
$\left(A_{1}, A_{2}, \ldots, A_{m-1}, B_{1}, B_{2}, \ldots, B_{n}, C_{1}, C_{2}, \ldots, C_{r}\right) \in \mathcal{A}_{\alpha}^{n, r}\left(\theta_{0}, a_{0}\right)$ and $f \in C((0, T) ; D) \cap$ $L_{1}(0, T ; D)$. Then the function

$$
z_{f}(t):=\int_{0}^{t} Z_{m-1}(t-s) f(s) d s
$$

is a unique solution to problem (9) and (10).

Proof. Arguing as in the proof of the inequality (8), we obtain at $\kappa<\alpha-1$ and $t \in[0, T]$,

$$
\begin{aligned}
\left\|V_{\mathcal{\kappa}}(t)\right\|_{\mathcal{L}(\mathcal{Z})} & \leq 2 c_{1} e^{\left(a_{0}+\varepsilon\right) t} \int_{r_{0}}^{+\infty} r^{\kappa-\alpha} e^{-r c_{2} t} d r+c_{1} r_{0}^{\kappa-\alpha} e^{\left(a_{0}+\varepsilon\right) t} \int_{-\theta_{0}+\varepsilon}^{\theta_{0}+\varepsilon} e^{t r_{0} \cos \varphi} d \varphi \\
& \leq \frac{2 c_{1} e^{\left(a_{0}+\varepsilon-r_{0} c_{2}\right) t} r_{0}^{\kappa-\alpha+1}}{\alpha-\kappa-1}+2 \pi c_{1} e^{\left(a_{0}+\varepsilon+r_{0}\right) t} r_{0}^{\kappa-\alpha} \leq C e^{\left(a_{0}+\varepsilon+r_{0}\right) t}
\end{aligned}
$$

for some $C>0$. Hence,

$$
\left\|\int_{0}^{t} V_{\mathcal{K}}(t-s) f(s) d s\right\|_{\mathcal{Z}} \leq C e^{\left(a_{0}+\varepsilon+r_{0}\right) t} \int_{0}^{t}\|f(s)\|_{\mathcal{Z}} d s \quad \text { for } \kappa<\alpha-1 .
$$

Also, for $\alpha \in(0,1]$ and $z_{0} \in D$, we have

$$
\begin{gathered}
V_{\alpha-1}(t) z_{0}=\frac{1}{2 \pi i} \int_{\Gamma_{\varepsilon, r_{0}}} \lambda^{\alpha-1} R_{\lambda} e^{\lambda t} z_{0} d \lambda \\
=\frac{1}{2 \pi i} \int_{\Gamma_{\varepsilon, r_{0}}} \frac{e^{\lambda t} d \lambda}{\lambda} z_{0}+\frac{1}{2 \pi i} \int_{\Gamma_{\varepsilon, r_{0}}} \lambda^{-1} R_{\lambda}\left(\sum_{j=1}^{m-1} \lambda^{\alpha-m+j} A_{j}+\sum_{l=1}^{n} \lambda^{\alpha_{l}} B_{l}+\sum_{s=1}^{r} \lambda^{\beta_{s}} C_{s}\right) z_{0} e^{\lambda t} d \lambda \\
=z_{0}+\frac{1}{2 \pi i} \int_{\Gamma} \lambda^{-1} R_{\lambda}\left(\sum_{j=1}^{m-1} \lambda^{\alpha-m+j} A_{j}+\sum_{l=1}^{n} \lambda^{\alpha_{l}} B_{l}+\sum_{s=1}^{r} \lambda^{\beta_{s}} C_{s}\right) z_{0} e^{\lambda t} d \lambda,
\end{gathered}
$$

while for $\lambda \in \Gamma_{\varepsilon, r_{0}}$,

$$
\left\|\lambda^{-1} R_{\lambda}\left(\sum_{j=1}^{m-1} \lambda^{\alpha-m+j} A_{j}+\sum_{l=1}^{n} \lambda^{\alpha_{l}} B_{l}+\sum_{s=1}^{r} \lambda^{\beta_{s}} C_{s}\right) z_{0}\right\|_{\mathcal{Z}} \leq \frac{c_{1}\left\|z_{0}\right\|_{D}}{|\lambda|^{1+\delta}},
$$

where $\delta=\min \left\{1, \alpha-\alpha_{l}: l=1,2, \ldots, n\right\}$. Therefore, for $t \in[0, T]$, we get

$$
\left\|V_{\alpha-1}(t) z_{0}\right\|_{\mathcal{Z}} \leq C e^{\left(a_{0}+\varepsilon+r_{0}\right) t}\left\|z_{0}\right\|_{D} \quad \text { for } \alpha \leq 1,
$$

and

$$
\left\|\int_{0}^{t} V_{\alpha-1}(t-s) f(s) d s\right\|_{\mathcal{Z}} \leq C e^{\left(a_{0}+\varepsilon+r_{0}\right) t} \int_{0}^{t}\|f(s)\|_{D} d s \quad \text { for } \alpha \leq 1 .
$$

Next, we define $f$ as zero for $t \in[T, \infty)$ and get the equalities

$$
\begin{gathered}
\mathfrak{L}\left[z_{f}\right](\mu)=\mathfrak{L}\left[Z_{m-1}\right](\mu) \mathfrak{L}[f](\mu)=R_{\mu} \mathfrak{L}[f](\mu), \\
\mathfrak{L}\left[J_{t}^{m-\alpha} z_{f}\right](\mu)=\mu^{\alpha-m} R_{\mu} \mathfrak{L}[f](\mu)
\end{gathered}
$$


and

$$
J_{t}^{m-\alpha} z_{f}(t)=\int_{0}^{t} V_{\alpha-m}(t-s) f(s) d s,
$$

therefore, $J_{t}^{m-\alpha} z_{f}(0)=0$, due to (12), if $\alpha \leq 1$, or (11) in other cases. By taking into account inequality (11) when $k=1,2, \ldots, m-2$, and inequality (12) at $k=m-1$, we obtain

$$
\begin{aligned}
& \mathfrak{L}\left[D_{t}^{\alpha-m+k} z_{f}\right](\lambda)=\mu^{\alpha-m+k} R_{\mu} \mathfrak{L}[f](\mu), \\
& D_{t}^{\alpha-m+k} z_{f}(t)=\int_{0}^{t} V_{\alpha-m+k}(t-s) f(s) d s
\end{aligned}
$$

and

$$
D_{t}^{\alpha-m+k} z_{f}(0)=0 .
$$

Note that according to corollaries 1 and 2, we have

$$
D_{t}^{\gamma} z_{f}(0)=0
$$

for $\gamma=\alpha_{l}-m_{l}+k, k \in\left\{0,1, \ldots, m_{l}-1\right\}$ and $l=1,2, \ldots, n$. Therefore,

$$
\begin{aligned}
\mathfrak{L}\left[D_{t}^{\alpha} z_{f}\right](\mu) & =\mu^{\alpha} R_{\mu} \mathfrak{L}[f](\mu) \\
& =\mathfrak{L}[f](\mu)+\left(\sum_{j=1}^{m-1} \mu^{\alpha-m+j} A_{j}+\sum_{l=1}^{n} \mu^{\alpha_{l}} B_{l}+\sum_{s=1}^{r} \mu^{-\beta_{s}} C_{s}\right) R_{\mu} \mathfrak{L}[f](\mu) \\
& =\mathfrak{L}[f](\mu)+\mathfrak{L}\left[\sum_{j=1}^{m-1} A_{j} D_{t}^{\alpha-m+j} z_{f}(t)+\sum_{l=1}^{n} B_{l} D_{t}^{\alpha_{l}} z_{f}(t)+\sum_{s=1}^{r} C_{s} J_{t}^{\beta_{s}} z_{f}(t)\right] .
\end{aligned}
$$

Acting by the inverse Laplace transform on both parts of this equality, we obtain equality (9) at $t \in(0, T)$, since $f \in C((0, T) ; D)$.

The proof of the uniqueness of a solution is the same as for the homogeneous equation.

Due to the linearity of the Equation (9) under study, we immediately get the following result.

Theorem 3. Let $m-1<\alpha \leq m \in \mathbb{N}, \alpha_{1}<\alpha_{2}<\cdots<\alpha_{n}<\alpha, m_{l}-1<\alpha_{l} \leq m_{l} \in \mathbb{N}$, $\alpha_{l}-m_{l} \neq \alpha-m, l=1,2, \ldots, n, \beta_{1}>\beta_{2}>\cdots>\beta_{r} \geq 0, A_{j} \in \mathcal{C l}(\mathcal{Z}), j=1,2, \ldots, m-1$, $B_{l} \in \mathcal{C l}(\mathcal{Z}), l=1,2, \ldots, n, C_{s} \in \mathcal{C l}(\mathcal{Z}), s=1,2, \ldots, r$,

$$
\left(A_{1}, A_{2}, \ldots, A_{m-1}, B_{1}, B_{2}, \ldots, B_{n}, C_{1}, C_{2}, \ldots, C_{r}\right) \in \mathcal{A}_{\alpha}^{n, r}\left(\theta_{0}, a_{0}\right),
$$

$z_{k} \in \mathcal{Z}, k=m^{*}, m^{*}+1, \ldots, m-1, f \in C((0, T) ; D) \cap L_{1}(0, T ; D)$. Then there exists a unique solution to problem (6) and (9), and it has the form

$$
z(t)=\sum_{p=m^{*}}^{m-1} Z_{p}(t) z_{p}+\int_{0}^{t} Z_{m-1}(t-s) f(s) d s .
$$

\section{Initial-Boundary Value Problems}

Let $J \subset\{1,2, \ldots, m-1\}$. Define polynomials $P_{1}, P_{2}^{j}, P_{3}^{l}, P_{4}^{s}$ by

$$
P_{1}(\lambda)=\sum_{p=0}^{v_{1}} a_{p} \lambda^{p}, \quad P_{2}^{j}(\lambda)=\sum_{p=0}^{v_{2}^{j}} b_{p}^{j} \lambda^{p}, \quad P_{3}^{l}(\lambda)=\sum_{p=0}^{v_{3}^{l}} c_{p}^{l} \lambda^{p} \quad \text { and } \quad P_{4}^{s}(\lambda)=\sum_{p=0}^{v_{4}^{s}} d_{p}^{s} \lambda^{p},
$$


where $a_{p}, b_{p}^{j}, c_{p}^{l}, d_{p}^{s} \in \mathbb{R}, a_{v_{1}} b_{{ }_{2}}^{j}{ }_{2}^{c} c_{v_{3}^{l}}^{l} d_{v_{4}^{s}}^{s} \neq 0, j \in J, l=1,2, \ldots, n, s=1,2, \ldots, r$ and the degree of at least one of the polynomials $P_{2}^{j}, P_{3}^{l}, P_{4}^{s}$ be greater than the degree of $P_{1}$. Put $P_{2}^{j} \equiv 0$ at $j \in\{1,2, \ldots, m-1\} \backslash J$.

Let $\Omega \subset \mathbb{R}^{d}$ be a boundary region with a smooth boundary $\partial \Omega$,

$$
(\mathfrak{A} u)(\xi)=\sum_{|q| \leq 2 \rho} a_{q}(\xi) \frac{\partial^{|q|} u(\xi)}{\partial \xi_{1}^{q_{1}} \partial \xi_{2}^{q_{2}} \ldots \partial \xi_{d}^{q_{d}}}, \quad a_{q} \in C^{\infty}(\bar{\Omega}),
$$

and

$$
\left(\mathfrak{B}_{\gamma} u\right)(\xi)=\sum_{|q| \leq \rho_{\gamma}} b_{\gamma q}(\xi) \frac{\partial^{|q|} u(\xi)}{\partial \xi_{1}^{q_{1}} \partial \xi_{2}^{q_{2}} \ldots \partial \xi_{d}^{q_{d}}}, \quad b_{\gamma q} \in C^{\infty}(\partial \Omega), \gamma=1,2, \ldots, \rho
$$

where $q=\left(q_{1}, q_{2}, \ldots, q_{d}\right) \in \mathbb{N}_{0}^{d},|q|=q_{1}+\cdots+q_{d}$ and the operator pencil $\mathfrak{A}, \mathfrak{B}_{1}, \mathfrak{B}_{2}, \ldots$, $\mathfrak{B}_{\rho}$ are regularly elliptic (see [21]). Assume that the operator $\mathfrak{A}_{1} \in \mathcal{C l}\left(L_{2}(\Omega)\right.$ ) has the domain

$$
D_{\mathfrak{A}_{1}}=H_{\left\{\mathfrak{B}_{\gamma}\right\}}^{2 \rho}(\Omega):=\left\{v \in H^{2 \rho}(\Omega): \mathfrak{B}_{\gamma} v(\xi)=0, \gamma=1,2, \ldots, \rho, \xi \in \partial \Omega\right\}
$$

and $\mathfrak{A}_{1} v:=\mathfrak{A} v$. Suppose that $\mathfrak{A}_{1}$ is self-adjoint, then the spectrum $\sigma\left(\mathfrak{A}_{1}\right)$ of $\mathfrak{A}_{1}$ is real and discrete (see [21]). Moreover, let the spectrum $\sigma\left(\mathfrak{A}_{1}\right)$ be bounded from the right and does not contain zero point, $\left\{\varphi_{k}: k \in \mathbb{N}\right\}$ is an orthonormal in the $L_{2}(\Omega)$ system of eigenfunctions of the operator $\mathfrak{A}_{1}$, numbered with respect to non-increasing of the corresponding eigenvalues $\left\{\lambda_{k}: k \in \mathbb{N}\right\}$, taking into account their multiplicity.

Take $h: \Omega \times(0, T) \rightarrow \mathbb{R}, 1<\alpha<2,0<\alpha_{1}<\alpha_{2}<\cdots<\alpha_{n}<\alpha, m_{l}-1<\alpha_{l} \leq$ $m_{l} \in \mathbb{N}, \alpha_{l}-m_{l} \neq \alpha-m, l=1,2, \ldots, n, \beta_{1}>\beta_{2}>\cdots>\beta_{r} \geq 0$. Determine by the set of these numbers the defect $m^{*} \in\{0,1\}$, choose the maximal degree $v_{0}$ of polynomials $P_{2}^{j}$, $P_{3}^{l}, P_{4}^{s}, j \in J, l=1,2, \ldots, n, s=1,2, \ldots, r,\left(\right.$ then $v_{0}>v_{1}$ ) and consider the initial-boundary value problem

$$
D_{t}^{\alpha-m+k} u(\xi, 0)=u_{k}(\xi), k=0,1 \text { or } k=1, \quad \xi \in \Omega,
$$

$$
\begin{aligned}
& \mathfrak{B}_{\gamma} \mathfrak{A}^{k} u(\xi, t)=0, \quad k=0,1, \ldots, v_{0}-1, \quad \gamma=1,2, \ldots, \rho, \quad(\xi, t) \in \partial \Omega \times(0, T), \\
& D_{t}^{\alpha} P_{1}(\mathfrak{A}) u(\xi, t)= \sum_{j \in J} P_{2}^{j}(\mathfrak{A}) D_{t}^{\alpha-m+j} u(\xi, t)+\sum_{l=1}^{n} P_{3}^{l}(\mathfrak{A}) D_{t}^{\alpha_{l}} u(\xi, t)+ \\
&+\sum_{s=1}^{r} P_{4}^{s}(\mathfrak{A}) J_{t}^{\beta_{s}} u(\xi, t)+h(\xi, t), \quad(\xi, t) \in \Omega \times(0, T) .
\end{aligned}
$$

Set

$$
\mathcal{X}=\left\{v \in H^{2 \rho v_{1}}(\Omega): \mathfrak{B}_{\gamma} \mathfrak{A}^{k} v(\xi)=0, k=0,1, \ldots, v_{1}-1, \gamma=1,2, \ldots, \rho, \xi \in \partial \Omega\right\},
$$

$\mathcal{Y}=L_{2}(\Omega), L=P_{1}(\mathfrak{A}) \in \mathcal{L}(\mathcal{X} ; \mathcal{Y}), M_{j}=P_{2}^{j}(\mathfrak{A}) \in \mathcal{C l}(\mathcal{X} ; \mathcal{Y})$ for $j=1,2, \ldots, m-1$, $N_{l}=P_{3}^{l}(\mathfrak{A}) \in \mathcal{C l}(\mathcal{X} ; \mathcal{Y})$ for $l=1,2, \ldots, n, S_{s}=P_{4}^{s}(\mathfrak{A}) \in \mathcal{C l}(\mathcal{X} ; \mathcal{Y})$ for $s=1,2, \ldots, r$. The domains of these closed operators are defined by polynomials, for example,

$$
D_{B_{l}}=\left\{v \in H^{2 \rho v_{3}^{l}}(\Omega): \mathfrak{B}_{\gamma} \mathfrak{A}^{k} v(\xi)=0, k=0,1, \ldots, v_{3}^{l}-1, \gamma=1,2, \ldots, \rho, \xi \in \partial \Omega\right\} .
$$

Therefore,

$$
D=\left\{v \in H^{2 \rho v_{0}}(\Omega): \mathfrak{B}_{\gamma} \mathfrak{A}^{k} v(\xi)=0, k=0,1, \ldots, v_{0}-1, \gamma=1,2, \ldots, \rho, \xi \in \partial \Omega\right\}
$$

is dense set in $\mathcal{X}$. 
Theorem 4. Let in the conditions of this section be $v_{0}>v_{1}, P_{1}\left(\lambda_{k}\right) \neq 0$ for all $k \in \mathbb{N}$, and the following conditions hold:

(i) In case of odd $v_{0}-v_{1}$, the inequalities $b_{v_{2}^{j}}^{j} / a_{v_{1}}>0$ at $v_{2}^{j}=v_{0}, c_{v_{3}^{l}}^{l} / a_{v_{1}}>0$ at $v_{3}^{l}=v_{0}$ and $c_{v_{4}^{s}}^{s} / a_{v_{1}}>0$ at $v_{4}^{s}=v_{0}$ hold;

(ii) In case of even $v_{0}-v_{1}$, the inequalities $b_{v_{2}^{j}}^{j} / a_{v_{1}}<0$ at $v_{2}^{j}=v_{0}, c_{v_{3}^{l}}^{l} / a_{v_{1}}<0$ at $v_{3}^{l}=v_{0}$ and $c_{v_{4}^{s}}^{s} / a_{v_{1}}<0$ at $v_{4}^{s}=v_{0}$ hold

(iii) $\beta_{s} \leq \alpha$ at $v_{4}^{s}=v_{0}$.

Then $\left(A_{1}, A_{2}, \ldots, A_{m-1}, B_{1}, B_{2}, \ldots, B_{n}, C_{1}, C_{2}, \ldots, C_{r}\right) \in \mathcal{A}_{\alpha}^{n, r}\left(\theta_{0}, a_{0}\right)$.

Proof. For $v \in D$,

$$
\left(\lambda^{\alpha} I-\sum_{j=1}^{m-1} \lambda^{\alpha-m+j} A_{j}-\sum_{l=1}^{n} \lambda^{\alpha_{l}} B_{l}-\sum_{s=1}^{r} \lambda^{-\beta_{s}} C_{s}\right) v=\sum_{k=1}^{\infty} \lambda^{\alpha} Q_{k}\left(\lambda, \lambda_{k}\right)\left\langle v, \varphi_{k}\right\rangle \varphi_{k},
$$

where,

$$
Q_{k}\left(\lambda, \lambda_{k}\right):=1-\sum_{j \in J} \lambda^{j-m} \frac{P_{2}^{j}\left(\lambda_{k}\right)}{P_{1}\left(\lambda_{k}\right)}-\sum_{l=1}^{n} \lambda^{\alpha_{l}-\alpha} \frac{P_{3}^{l}\left(\lambda_{k}\right)}{P_{1}\left(\lambda_{k}\right)}-\sum_{s=1}^{r} \lambda^{-\beta_{s}-\alpha} \frac{P_{4}^{s}\left(\lambda_{k}\right)}{P_{1}\left(\lambda_{k}\right)} .
$$

It is evident, that for a fixed $k \in \mathbb{N}, \lim _{|\lambda| \rightarrow \infty} Q_{k}\left(\lambda, \lambda_{k}\right)=1$. Choose a sufficiently large $N \in \mathbb{N}$ so large $R>0$, that $Q_{k}\left(\lambda, \lambda_{k}\right) \geq 1 / 2$ for $k=1,2, \ldots, N$ and $|\lambda|>R$. Since $\lim _{k \rightarrow \infty} \lambda_{k}=-\infty$, letting $k \rightarrow \infty$, we have

$$
\begin{gathered}
\frac{P_{2}^{j}\left(\lambda_{k}\right)}{P_{1}\left(\lambda_{k}\right)} \sim a_{v_{1}}^{-1} b_{v_{2}^{j}}^{j} \lambda_{k}^{v_{2}^{j}-v_{1}} \quad \text { for } j \in J, \\
\frac{P_{3}^{l}\left(\lambda_{k}\right)}{P_{1}\left(\lambda_{k}\right)} \sim a_{v_{1}}^{-1} c_{v_{3}^{l}}^{l} \lambda_{k}^{v_{3}^{l}-v_{1}} \quad \text { for } l=1,2, \ldots, n,
\end{gathered}
$$

and

$$
\frac{P_{4}^{s}\left(\lambda_{k}\right)}{P_{1}\left(\lambda_{k}\right)} \sim a_{v_{1}}^{-1} d_{v_{4}^{s}}^{s} \lambda_{k}^{v_{4}^{s}-v_{1}} \quad \text { for } s=1,2, \ldots, r
$$

Hence for a fixed $\lambda$ as $k \rightarrow \infty$, we get

$$
\begin{gathered}
Q_{k}\left(\lambda, \lambda_{k}\right) \sim 1-\sum_{v_{2}^{j}=v_{0}} \lambda^{j-m} a_{v_{1}}^{-1} b_{v_{2}^{j}}^{j} \lambda_{k}^{v_{0}-v_{1}} \\
-\sum_{v_{3}^{l}=v_{0}} \lambda^{\alpha_{l}-\alpha} a_{v_{1}}^{-1} c_{v_{3}^{l}}^{l} \lambda_{k}^{v_{0}-v_{1}}-\sum_{v_{4}^{s}=v_{0}} \lambda^{-\beta_{s}-\alpha} a_{v_{1}}^{-1} d_{v_{4}^{s}}^{s} \lambda_{k}^{v_{0}-v_{1}} \\
=\left|\lambda_{k}\right|^{v_{0}-v_{1}}\left[\left|\lambda_{k}\right|^{v_{1}-v_{0}}-\operatorname{sign} \lambda_{k}^{v_{0}-v_{1}}\left(\sum_{v_{2}^{j}=v_{0}} \lambda^{j-m} a_{v_{1}}^{-1} b_{v_{2}^{j}}^{j}\right.\right. \\
\left.\left.+\sum_{v_{3}^{l}=v_{0}} \lambda^{\alpha_{l}-\alpha} a_{v_{1}}^{-1} c_{v_{3}^{l}}^{l}+\sum_{v_{4}^{s}=v_{0}} \lambda^{-\beta_{s}-\alpha} a_{v_{1}}^{-1} d_{v_{4}^{s}}^{s}\right)\right] .
\end{gathered}
$$


For sufficiently large $k, \lambda_{k}<0$ due to properties of the spectrum of $\mathfrak{A}_{1}$, therefore, due to conditions (i) and (ii) of this theorem all coefficients at the degrees of $\lambda$ in the last expression are negative. Hence at $\lambda>0$ and for sufficiently large $k Q\left(\lambda, \lambda_{k}\right)>0$. Take a sufficiently large $a_{0}>R$ and $\theta \in(\pi / 2, \pi / \alpha)$, so that the ball $B_{R}:=\{\lambda \in \mathbb{C}:|\lambda| \leq R\}$ lies outside the sector $S_{\theta_{0}, a_{0}}$. Then for $\lambda \in S_{\theta_{0}, a_{0}} \backslash \mathbb{R}$ subject to condition (iii) of this theorem,

$$
\left|\arg \left[\operatorname{sign} \lambda_{k}^{v_{0}-v_{1}}\left(\sum_{v_{2}^{j}=v_{0}} \lambda^{j-m} a_{\nu_{1}}^{-1} b_{v_{2}^{j}}^{j}+\sum_{v_{3}^{l}=v_{0}} \lambda^{\alpha_{l}-\alpha} a_{v_{1}}^{-1} c_{v_{3}^{l}}^{l}+\sum_{v_{4}^{s}=v_{0}} \lambda^{-\beta_{s}-\alpha} a_{v_{1}}^{-1} d_{v_{4}^{s}}^{s}\right)\right]\right| \in(0, \pi),
$$

therefore, $Q_{k}\left(\lambda, \lambda_{k}\right) \neq 0$. If $\partial S_{\theta_{0}, a_{0}}$ contains zeros (with respect to $\lambda$ ) of functions $Q_{k}\left(\lambda, \lambda_{k}\right)$ at some $k \in \mathbb{N}$, beginning with $N+1$, make the shift of $a_{0}$ on 1 to the right and go back to the previous designation $a_{0}$. Now due to the continuity of the functions $Q_{k}\left(\lambda, \lambda_{k}\right)$ with respect to $\lambda$ we can state that for $\lambda \in S_{a_{0}, \theta_{0}}$, we have $\left|Q_{k}\left(\lambda, \lambda_{k}\right)\right| \geq c>0$.

Thus, for every $\lambda \in S_{\theta_{0}, a_{0}}$, we obtain

$$
\left\|\left(\lambda^{\alpha} I-\sum_{j=1}^{m-1} \lambda^{\alpha-m+j} A_{j}-\sum_{l=1}^{n} \lambda^{\alpha_{l}} B_{l}-\sum_{s=1}^{r} \lambda^{-\beta_{s}} C_{s}\right) v\right\|_{\mathcal{X}} \geq c|\lambda|^{\alpha}\|v\|_{\mathcal{X}}
$$

consequently, there exists the inverse operator

$$
R_{\lambda}:=\left(\lambda^{\alpha} I-\sum_{j=1}^{m-1} \lambda^{\alpha-m+j} A_{j}-\sum_{l=1}^{n} \lambda^{\alpha_{l}} B_{l}-\sum_{s=1}^{r} \lambda^{-\beta_{s}} C_{s}\right)^{-1} \in \mathcal{L}(\mathcal{X}),
$$

and for all $\lambda \in S_{\theta_{0}, a_{0}}$

$$
\left\|R_{\lambda}\right\| \leq \frac{C}{|\lambda|^{\alpha-1}|\lambda-a|}
$$

For $v \in D$ and $\lambda \in S_{\theta_{0}, a_{0}}$, we have

$$
\begin{aligned}
& \left\|R_{\lambda} \cdot\left(I-\sum_{j=p+1}^{m-1} \lambda^{j-m} A_{j}\right) v\right\|_{\mathcal{X}}^{2} \\
& \leq \sum_{k=1}^{\infty} \frac{2\left(1+\sum_{j=p+1}^{m-1}|\lambda|^{2(j-m)} \frac{\left|P_{2}^{j}\left(\lambda_{k}\right)\right|^{2}}{\left|P_{1}\left(\lambda_{k}\right)\right|^{2}}\right)\left|\left\langle v, \varphi_{k}\right\rangle\right|^{2}}{|\lambda|^{2 \alpha}\left|Q\left(\lambda, \lambda_{k}\right)\right|^{2}} \\
& \leq \sum_{k=1}^{\infty} \frac{C_{1}\left|\lambda_{k}\right|^{2\left(v_{0}-v_{1}\right)}\left(\left|\lambda_{k}\right|^{-2\left(v_{0}-v_{1}\right)}+\sum_{v_{2}^{j}=v_{0}}\left|a_{v_{1}}\right|^{-2}\left|b_{v_{2}^{j}}\right|^{2}|\lambda|^{2(j-m)}\right)\left|\left\langle v, \varphi_{k}\right\rangle\right|^{2}}{|\lambda|^{2 \alpha}\left|\lambda_{k}\right|^{2\left(v_{0}-v_{1}\right)}\left|Q_{1}\left(\lambda, \lambda_{k}\right)\right|^{2}} \\
& \leq \sum_{k=1}^{\infty} \frac{C_{2}\left|\left\langle v, \varphi_{k}\right\rangle\right|^{2}}{|\lambda|^{2(\alpha-1)}|\lambda-a|^{2}} \leq \frac{C_{2}\|v\|_{\mathcal{X}}^{2}}{|\lambda|^{2(\alpha-1)}|\lambda-a|^{2}},
\end{aligned}
$$

and

$$
Q_{1}\left(\lambda, \lambda_{k}\right)=\lambda_{k}^{-\left(v_{0}-v_{1}\right)}-\sum_{v_{2}^{j}=v_{0}} \lambda^{-m+j} a_{v_{1}}^{-1} b_{v_{2}^{j}}^{j}-\sum_{v_{3}^{l}=v_{0}} \lambda^{\alpha_{l}-\alpha} a_{v_{1}}^{-1} c_{v_{3}^{l}}^{l}-\sum_{v_{4}^{s}=v_{0}} \lambda^{-\beta_{s}-\alpha} a_{v_{1}}^{-1} d_{v_{4}^{s}}^{s} .
$$

We take into account, that $\lambda_{k} \neq 0$ for all $k \in \mathbb{N}$, therefore, due to the pointiness of the spectrum $\left\{\lambda_{k}\right\}$ there exists $\min _{k \in \mathbb{N}}\left|\lambda_{k}\right|>0$.

Remark 5. For the case of $v_{0} \leq v_{1}$, a similar result in the absence of constraints (i)-(iii) from Theorem 4 is obtained in [8]. 
Due to Theorem 3 for arbitrary $u_{0}, u_{1} \in \mathcal{X}$, or only $u_{1} \in \mathcal{X}$, depending on the defect value, under the condition $L^{-1} h \in C((0, T) ; D) \cap L_{1}(0, T ; D)$ there exists a unique solution to problem (13)-(15).

Finally, a nontrivial example illustrating Theorem 4 is given.

Example 1. Take $\alpha=7 / 4, m=2, n=1, r=1, \alpha_{1}=4 / 5, \beta_{1}=5, P_{1}(\lambda)=\lambda, v_{1}=1$, $P_{2}^{1}(\lambda) \equiv 0, J=\varnothing, P_{3}^{1}(\lambda)=c_{0}+c_{1} \lambda+c_{2} \lambda^{2}, v_{3}=2, P_{4}^{1}(\lambda)=d_{0}+d_{1} \lambda, v_{4}=1, v_{0}:=$ $\max \left\{v_{3}, v_{4}\right\}=2, d=1, \Omega=(0, \pi), \rho=1, \mathfrak{A} u=\frac{\partial^{2} u}{\partial \xi^{2}}$ and $\mathfrak{B}_{1}=I$. Then $\underline{m}=0, \bar{m}=1$, $m^{*}=1$, and problem (13)-(15) has the form

$$
D_{t}^{7 / 4} \frac{\partial^{2} u}{\partial \xi^{2}}(\xi, t)=\left(c_{0}+c_{1} \frac{\partial^{2}}{\partial \xi^{2}}+c_{2} \frac{\partial^{4}}{\partial \xi^{4}}\right) D_{t}^{4 / 5} u(s, t)+\left(d_{0}+d_{1} \frac{\partial^{2}}{\partial \xi^{2}}\right) J_{t}^{5} u(\xi, t)
$$

for $(\xi, t) \in(0, \pi) \times \mathbb{R}_{+}$,

$$
u(0, t)=u(\pi, t)=\frac{\partial^{2} u}{\partial s^{2}}(0, t)=\frac{\partial^{2} u}{\partial s^{2}}(\pi, t)=0 \quad \text { for } t \in \mathbb{R}_{+},
$$

and

$$
D^{3 / 4} u(\xi, 0)=u_{1}(\xi) \quad \text { for } \xi \in(0, \pi) .
$$

It is solvable at $c_{2}>0$ by Theorem $4(i)$, since $v_{0}-v_{1}=1$ is odd, $v_{3}=v_{0}, v_{4}<v_{0}$. Here the problem does not contain the initial condition for $J^{1 / 4} u(\xi, 0)$, since $m^{*}=1$ and the equality $J^{1 / 4} u(\xi, 0) \equiv 0$ is necessarily fulfilled.

\section{Conclusions}

For a linear equation with several Riemann-Liouville derivatives and with constant coefficients, the authors of this work showed that the set of orders of derivatives from the equation determines the defect of the Cauchy-type problem, which determines the number of lower-order initial conditions that must be excluded from the statement of the problem for its solvability. In this paper, the incomplete Cauchy problem is considered for linear multi-term equations solved with respect to the highest derivative in Banach spaces with fractional Riemann-Liouville derivatives and with linear closed operators at them. A class of tuples of operators $\mathcal{A}_{\alpha}^{n, r}\left(\theta_{0}, a_{0}\right)$ is introduced, generalizing to the case of several operators a class of generators of analytic in a sector resolving families of operators, called sectorial operators. Using the Laplace transform technique, it is proved that the incomplete Cauchy-type problem for the multi-term equation with a tuple of operators from $\mathcal{A}_{\alpha}^{n, r}\left(\theta_{0}, a_{0}\right)$ has a unique classical solution. Some new existence and uniqueness theorems for solutions are presented explicitly and the analyticity of the solutions of the homogeneous equations are also shown. Our abstract results are used in the study of a class of initial boundary value problems for multi-term equations with Riemann-Liouville derivatives in time and with polynomials of a self-adjoint elliptic differential operator with respect to spatial variables. In contrast to our earlier result, the consideration of the equation with unbounded operators allowed us to investigate the case when the highest of the degrees of the polynomials at lower time-fractional derivatives is greater than the degree of the polynomial at the highest derivative.

Author Contributions: Conceptualization, V.E.F. and W.-S.D.; methodology, V.E.F. and W.-S.D.; software, M.M.T.; validation, V.E.F. and M.M.T.; formal analysis, M.M.T.; investigation, V.E.F., W.-S.D. and M.M.T.; resources, W.-S.D.; data curation, V.E.F. and W.-S.D.; writing-original draft preparation, V.E.F. and M.M.T.; writing—review and editing, V.E.F. and W.-S.D.; visualization, M.M.T.; supervision, V.E.F. and W.-S.D.; project administration, V.E.F. and W.-S.D.; funding acquisition, V.E.F. and W.-S.D. All authors have read and agreed to the published version of the manuscript. 
Funding: The first and the third authors is partially supported by the Russian Foundation for Basic Research and Vietnam Academy of Science and Technology, grant number 21-51-54003, and by the grant of the President of the Russian Federation to support leading scientific schools, project number NSh-2708.2022.1.1. The second author is partially supported by grant no. MOST 110-2115-M-017-001 of the Ministry of Science and Technology of the Republic of China.

Institutional Review Board Statement: Not applicable.

Informed Consent Statement: Not applicable.

Data Availability Statement: Not applicable.

Acknowledgments: The authors wish to express their hearty thanks to the anonymous referees for their valuable suggestions and comments.

Conflicts of Interest: The authors declare no conflict of interest.

\section{References}

1. Luchko, Y.F.; Srivastava, H.M. The exact solution of certain differential equations of fractional order by using operational calculus. Comput. Math. Appl. 1995, 29, 73-85. [CrossRef]

2. Hadid, S.B.; Luchko, Y.F. An operational method for solving fractional differential equations of an arbitrary real order. Panam. Math. J. 1996, 6, 57-73.

3. Ozturk, I. On the theory of fractional differential equation. Rep. Adyg. (Circassian) Int. Acad. Sci. 1998, 3, 35-39.

4. Nakhushev, A.M. Fractional Calculus ant Its Applications; Fizmatlit: Moscow, Russia, 2003. (In Russian)

5. Kilbas, A.A.; Srivastava, H.M.; Trujillo, J.J. Theory and Applications of Fractional Differential Equations; Elsevier Science Publishing: Amsterdam, The Netherlands; Boston, MA, USA; Heidelberg, Germany, 2006.

6. Pskhu, A.V. Initial-value problem for a linear ordinary differential equation of noninteger order. Sb. Math. 2011, $202,571-582$. [CrossRef]

7. Bhrawy, A.H.; Tharwat, M.M.; Yildirim, A. A new formula for fractional integrals of Chebyshev polynomials: Application for solving multi-term fractional differential equations. Appl. Math. Model. 2013, 37, 4245-4252. [CrossRef]

8. Fedorov, V.E.; Turov, M.M. The defect of a Cauchy type problem for linear equations with several Riemann-Liouville derivatives. Sib. Math. J. 2021, 62, 925-942. [CrossRef]

9. Bajlekova, E.G. Fractional Evolution Equations in Banach Spaces. Ph.D. Thesis, Eindhoven University of Technology, Eindhoven, The Netherlands, 2001.

10. Romanova, E.A.; Fedorov, V.E. Resolving operators of a linear degenerate evolution equation with Caputo derivative. The sectorial case. Math. Notes NEFU 2016, 23, 58-72.

11. Fedorov, V.E.; Avilovich, A.S. A Cauchy type problem for a degenerate equation with the Riemann-Liouville derivative in the sectorial case. Sib. Math. J. 2019, 60, 359-372. [CrossRef]

12. Fedorov, V.E.; Avilovich, A.S.; Borel, L.V. Initial problems for semilinear degenerate evolution equations of fractional order in the sectorial case. Springer Proc. Math. Stat. 2019, 292, 41-62.

13. Avilovich, A.S.; Gordievskikh, D.M.; Fedorov, V.E. Issues of unique solvability and approximate controllability for linear fractional order equations with a Hölderian right-hand side. Chelyabinsk Phys. Math. J. 2020, 5, 5-21.

14. Fedorov, V.E.; Nagumanova, A.V.; Avilovich, A.S. A class of inverse problems for evolution equations with the Riemann-Liouville derivative in the sectorial case. Math. Methods Appl. Sci. 2021, 44, 11961-11969. [CrossRef]

15. Fedorov, V.E.; Avilovich, A.S. Semilinear fractional-order evolution equations of Sobolev type in the sectorial case. Complex Var. Elliptic Equ. 2021, 66, 1108-1121. [CrossRef]

16. Fedorov, V.E.; Kostić, M. On a class of abstract degenerate multi-term fractional differential equations in locally convex spaces. Eurasian Math. J. 2018, 9, 33-57. [CrossRef]

17. Fedorov, V.E.; Boyko, K.V.; Phuong, T.D. Initial value problems for some classes of linear evolution equations with several fractional derivatives. Math. Notes NEFU 2021, 28, 85-104.

18. Podlubny, I. Fractional Differential Equations; Academic Press: Boston, MA, USA, 1999.

19. Yosida, K. Functional Analysis; Springer: Berlin/Heidelberg, Germany, 1965.

20. Kato, K. Perturbation Theory for Linear Operators; Springer: Berlin/Heidelberg, Germany, 1966.

21. Triebel, H. Interpolation Theory. Function Spaces. Differential Operators; Deutscher Verlag des Wissenschaften: Berlin, Germany, 1978. 